\title{
UNSTEADY FLOW INDUCED BY A WITHDRAWAL POINT BENEATH A FREE SURFACE
}

\author{
T. E. STOKES ${ }^{1}$, G. C. HOCKING ${ }^{\curvearrowleft 2}$ and L. K. FORBES 3
}

(Received 30 July, 2004; revised 22 July, 2005)

\begin{abstract}
The unsteady axisymmetric withdrawal from a fluid with a free surface through a point sink is considered. Results both with and without surface tension are included and placed in context with previous work. The results indicate that there are two critical values of withdrawal rate at which the surface is drawn directly into the outlet, one after flow initiation and the other after the flow has been established. It is shown that the larger of these values corresponds to the point at which steady solutions no longer exist.
\end{abstract}

\section{Introduction}

The study of withdrawal from stratified fluids such as those found in water storage reservoirs, cooling ponds or solar ponds is important for both management planning and water quality [17]. Our concern here is with the particular case in which the stratification consists of two layers of different density and in particular where the upper layer is air and the outlet is a point sink. Thus it is like the problem of water flowing out of a bath tub. The equations are identical to the case of two layers of different temperature (for example) in which the upper layer is assumed to be stagnant [33] and where gravity, $g$, is replaced by a reduced gravity (moderated by the density difference), that is, $g^{\prime}=(\Delta \rho / \rho) g$, where $\Delta \rho$ is the density difference and $\rho$ is some reference density [12].

It is known that in this situation all of the withdrawn fluid comes from the water until a critical flow rate is reached after which air begins to be entrained into the hole. In the bathtub example, this usually means the formation of a vortex, see for

\footnotetext{
'Department of Mathematics, University of Waikato, Hamilton, New Zealand.

${ }^{2}$ School of Engineering Science, Murdoch University, Murdoch, WA 6150, Australia; e-mail:

g.hocking@murdoch.edu.au.

${ }^{3}$ School of Mathematics and Physics, University of Tasmania, GPO Box 252-37, Hobart, TAS 7001,

Australia.

(C) Australian Mathematical Society 2005, Serial-fee code 1446-1811/05
} 
example Forbes and Hocking [4]. Experiments (performed in the two-layer fluid situation) provide a range of different critical values (at which the transitions occur) and flow patterns for the two-layer flow, depending on the geometry, density, type of withdrawal (line or point sink), flow rate, initial configuration and a range of other factors $[7,9,11,16,18,19,20,26,31]$.

The main parameter that controls such flows into a point sink is the Froude number

$$
F=\left(\frac{m^{2}}{16 \pi^{2} g H^{5}}\right)^{1 / 2},
$$

where $m$ is the sink strength, $H$ is the sink depth beneath the undisturbed free surface and $g$ is gravitational acceleration. Note that $m$ is the total flux into the point sink, so increasing $F$ means either increasing flow rate or decreasing the height of the fluid above the sink.

In the analogous line sink case the equivalent parameter is

$$
F_{2 D}=\left(\frac{\mu^{2}}{4 \pi^{2} g H^{3}}\right)^{1 / 2},
$$

where $\mu$ is the withdrawal rate per unit width. In that case, steady-state flows have been found to consist of two major types, cusp-like solutions $[1,10,11,24,28,30]$ in which the free surface is drawn down into a cusp above the sink, and stagnation point flows $[3,13,22,28,30]$ in which it rises to a single stagnation point above the sink. The unsteady version of this problem was considered in detail by Stokes et al. [26] and Tyvand [29]. In summary, it has been found that at low values of $F_{2 D}$ there exist steady-state flows containing a stagnation point on the free surface (or interface). As $F_{2 D}$ rises (corresponding to higher withdrawal rate or shallower water depth), there is a transition to a regime where no steady states exist. This unsteady regime ends when a cusp solution forms at the Froude number just below the transition to a supercritical flow in which the second layer is also drawn into the outlet $[12,14]$. Unsteady results also appear to indicate that there are two critical values of flow. It would seem that if the value of $F_{2 D}$ is large enough during initiation of the flow, the interface will immediately draw down, but if not, it must wait until a higher value (assuming $F_{2 D}$ gradually increases as time proceeds corresponding to a drop in the water level).

Here we consider the three-dimensional analogue of this problem in which there is a submerged point sink and the flow is radially symmetric. Calculations in three dimensions are much more difficult than those in two dimensions, illustrated by the relative scarcity of such solutions. To put this work in context we note that for axisymmetric, subcritical flows the only existing known steady solutions are of the stagnation point type in infinite depth [2] and in finite depth [6, 15]. Supercritical flows including rotation can be found in Forbes and Hocking [4], while unsteady 
computations are given in Tyvand [29] and Xue and Yue [32]. The steady, subcritical solutions all develop a standing wave close to $r=0$ that rises up to be a secondary stagnation line as the Froude number increases to the limiting value, $F \approx 0.51$. Note that this Froude number is defined differently (by a factor of $4 \pi$ ) to that used in Forbes and Hocking [2]. Beyond this, no steady states of any kind have been found. No cusp solutions or general two-layer flows have been obtained for these axisymmetric flows, although some similar flows have been computed $[4,5]$.

It is the aim of this paper to investigate the axisymmetric case more deeply using numerical techniques to analyse the unsteady flow. The numerical method is validated by considering the first terms of relevant small time expansions, using an approach similar to that of Miloh and Tyvand [21]. Comparison with asymptotic and numerically computed steady states provide further validation of the method.

Evidence will be given to suggest that, as in the line sink case [26], there are two critical drawdown values of the Froude number. One of these is related to the initiation of the flow, and the other to the case of a fully developed flow subject to an increase in Froude number (such as when the level of the water drops). These results may help to explain the diversity in critical values obtained in experimental results $[7,9,11,16,18,19,20,31]$.

In addition, the flow with surface tension acting on the free surface will be considered. These results will also be compared with earlier steady results [2]. It turns out that the inclusion of surface tension on the free surface has a stabilising effect that improves the distinction between different types of solutions.

\section{Problem formulation}

In this paper we consider the axisymmetric, unsteady, irrotational flow of an inviscid, incompressible, (infinitely) deep fluid into a point sink beneath a free surface. The assumption of radial symmetry means the problem can be reduced to finding the free surface profile as a function of radial distance from the origin.

2.1. Model equations A point sink is initially situated at a depth $H$ beneath the undisturbed level of the free surface and has strength $m$. The free surface is assumed to have surface tension $S$, although this parameter will often be set to zero. The problem is to find the velocity potential $\Phi(r, z, t)$ within the fluid beneath the free surface with kinematic and dynamic conditions on the surface and subject to the existence of the point sink at $z=-H$.

Nondimensionalising the potential, time and length with respect to $(m / 4 \pi H)$, $\left(4 \pi H^{3} / m\right)$ and $H$ respectively, the problem becomes that of solving

$$
\nabla^{2} \phi=0, \quad z<\eta(r, t), \quad(r, z) \neq(0,-1),
$$


subject to

and

$$
\phi_{t}+\frac{1}{2}\left(u^{2}+v^{2}\right)+F^{-2} \eta+\frac{F^{-2} \beta\left(\eta_{r r}+\eta_{r} / r\right)}{\left(1+\eta_{r}^{2}\right)^{3 / 2}}=0 \quad \text { on } z=\eta(r, t)
$$

$$
\eta_{t}+\phi_{r} \eta_{r}-\phi_{z}=0 \text { on } z=\eta(r, t)
$$

with the extra condition that

$$
\phi \rightarrow\left(r^{2}+(z+1)^{2}\right)^{-1 / 2} \text { as }(r, z) \rightarrow(0,-1)
$$

since the sink is situated at $z=-1$ in the new system, and $z=\eta(r, t)$ is the location of the free surface. Equation (2.3) is the dynamic condition and Equation (2.4) is the kinematic condition. The important quantities are then the Froude number, $F$, and the nondimensional surface tension, $\beta=S /\left(g H^{2}\right)$.

Figure 1 shows the flow configuration including the results of actual calculations of free surface shape at two different times $(t=0.18,0.32)$ when $F=0.3$ and $\beta=0$.

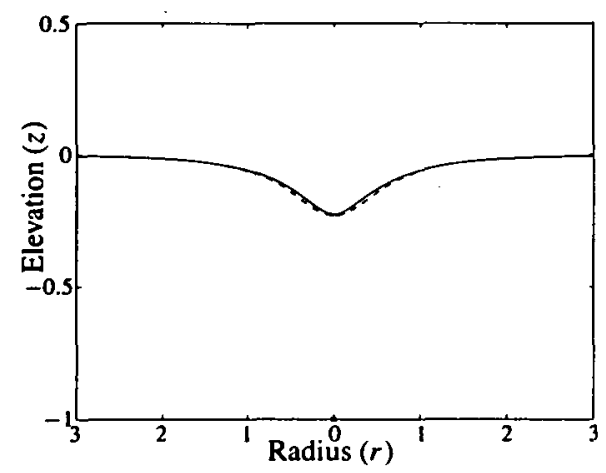

(a) $t=0.18$

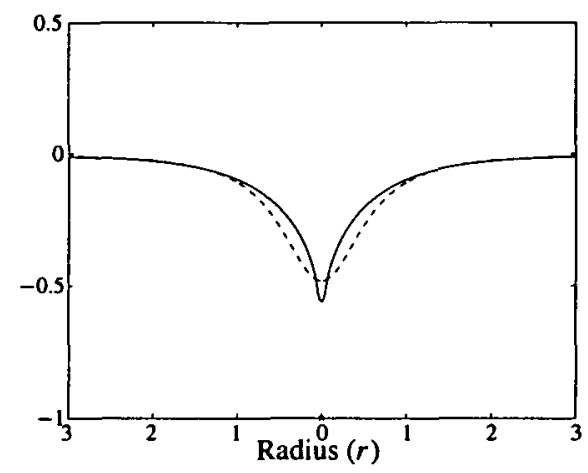

(b) $t=0.32$

FIGURE 1. Free surface profiles for $F=0.3, \beta=0$; numerical simulations -, small time expansion - The sink is located at $z=-1$.

It remains to consider the initial conditions on the flow. If the fluid is at rest it will initially have a horizontal surface, that is, $\eta(r, 0)=0$. However, there are several possible initial conditions on $\phi(r, z, t)$.

Miloh and Tyvand [21] and Xue and Yue [32] chose to use the condition that the potential on the free surface (interface) is constant, so that

$$
\phi(r, 0,0)=0, \quad r>0,
$$

on the basis that this is the case before the sink flow is initiated. This has the effect of imposing a purely vertical initial motion on the free surface, as if an image source 
were placed at $z=1$. Their results can be found in the cited works, and so we will only consider them as a comparison to the present results.

An alternative initial condition (see Stokes et al. [26]) is obtained by considering the initial flow to be the potential due to the line sink. It is known that in stratified flows, the initial flow is potential until buoyancy takes effect [33]. Thus along the free surface we obtain the potential due to the sink flow, that is,

$$
\phi(r, 0,0)=\left(r^{2}+1\right)^{-1 / 2}, \quad r>0 .
$$

These two initial conditions lead to very similar qualitative behaviour. Initial pulse amplitudes on the free surface are of the order of the Froude number, $O(F)$. The condition of Miloh and Tyvand [21] leads to slightly higher amplitudes but retains this general dependence. Drawdown occurs at different, but similar, values and the general wave behaviour is the same.

A third possible initial condition is one for which the vertical motion of the interface is suppressed, so that

$$
\phi_{z}(r, 0,0)=0, r>0 \text {. }
$$

This is equivalent to placing a rigid lid on the surface, starting the withdrawal and then removing the lid once the flow is established. This leads to disturbances of order $O\left(F^{2}\right)$, a much smaller disturbance in general than the other two cases. Stokes et al. [26] speculated that this would correspond to a situation in which the flux was increased very slowly from zero up to the value of interest. The initial condition is equivalent to initially assuming that

$$
\phi(r, z, 0)=\left(r^{2}+(z+1)^{2}\right)^{-1 / 2}+\left(r^{2}+(z-1)^{2}\right)^{-1 / 2}, \quad r>0,
$$

which can be viewed as placing an "image sink" of equal magnitude as the submerged sink and directly above it, one unit above the interface.

The numerical experiments shortly described show that the choice of initial condition continues to have an effect even if the flow rate is increased fairly rapidly (by increasing $F$ ) from zero to some constant value. However, if the flow rate is increased from zero to a small value, then allowed to settle, and then ramped up again, the flow behaves completely differently, and becomes independent of the initial condition, that is, (2.6) or (2.5), and behaves like the simulations using (2.7)! Since in many applications this would be the real situation (for example in a reservoir, withdrawal is rarely zero, but moves from low to high and high to low depending on the time of day), it is of great interest to study this situation. 


\section{Small time expansions}

In this section we compute the first two terms in the small time expansions for the two situations of interest to us. The "single sink" case, with initial condition (2.6) has a sink beneath the surface at $(0,-1)$, while the "image sink" case has this as well as an image sink situated at $(0,1)$ outside of the fluid domain, in order to satisfy condition (2.7). These calculations, together with the results given in Miloh and Tyvand [21] using (2.5), provide a useful study of the initial behaviour of the free surface and a check on the numerical method over relatively short times.

\subsection{Series solution in the general case We seek solutions of the form}

$$
\eta(r, t)=t \eta_{1}(r)+t^{2} \eta_{2}(r)+\cdots
$$

and

$$
\phi(r, z, t)=\phi_{0}(r, z)+t \phi_{1}(r, z)+t^{2} \phi_{2}(r, z)+\cdots .
$$

Expanding (3.2) in $z$ about $z=0$ gives

$$
\begin{aligned}
\phi(r, z, t)= & \phi_{0}(r, 0)+t \phi_{1}(r, 0)+t^{2} \phi_{2}(r, 0)+\cdots \\
& +z \phi_{0 z}(r, 0)+z t \phi_{1 z}(r, 0)+z t^{2} \phi_{2 z}(r, 0)+\cdots \\
& +\frac{1}{2} z^{2} \phi_{0 z z}(r, 0)+\frac{1}{2} z^{2} t \phi_{12 z}(r, 0)+\frac{1}{2} z^{2} t^{2} \phi_{2 z z}(r, 0)+\cdots
\end{aligned}
$$

Equation (3.1) is substituted into this expression and into the equivalent expressions for $\phi_{r}, \phi_{r}$ and $\phi_{z}$, and the result is incorporated into (2.2) and (2.3). Equating coefficients of powers of $t$ up to degree zero in (2.2) and degree one in (2.3), we find $\phi_{t}=\phi_{1}$, $\phi_{r}=\phi_{0 r}$ and $\phi_{z}=\phi_{0 z}+t \phi_{1 z}+z \phi_{0 z z}$, where all are evaluated on the line $z=0$ at time $t=0$. Now substituting (3.1) into each of these three equations and substituting the result into (2.2), collecting powers of $t$ and equating the constant term and coefficients of $t$ to zero we obtain the dynamic condition

$$
\phi_{1}+\frac{1}{2}\left(\phi_{0 r}^{2}+\phi_{0 z}^{2}\right)=0, \quad \text { on } z=0 .
$$

Similarly, substituting into (2.3) and equating coefficients to zero gives the kinematic condition at first order as

$$
\eta_{1}-\phi_{0 z}=0, \quad \text { on } z=0
$$

and at second order,

$$
-\phi_{1 z}-\eta_{1} \phi_{0 z z}+\eta_{1 r} \phi_{0 r}+2 \eta_{2}=0, \quad \text { on } z=0 .
$$

Thus it is possible to determine $\eta_{1}$ from the kinematic condition (3.4), $\phi_{1}$ from the dynamic condition (3.3) providing $\phi_{0}$ is known, and then $\eta_{2}$ from the second-order 
kinematic condition (3.5). Notice that $F$ and $\beta$ do not appear in these terms, so that gravity and surface tension do not play a role in the early part of the flow.

3.2. Single sink case Here we assume, consistent with initial condition (2.6), that

$$
\phi_{0}(r, z)=\frac{1}{\left(r^{2}+(z+1)^{2}\right)^{1 / 2}},
$$

representing a point sink immersed in the fluid at $(0,-1)$. (Recall that the fluid-free surface is at $z=0$ initially.) The first-order kinematic condition, (3.4), then gives

$$
\eta_{1}(r)=\phi_{0 z}=-\frac{1}{\left(r^{2}+1\right)^{3 / 2}} .
$$

Figure 2 shows a plot (solid line) of a cross-section of this shape. Thus a dip forms and grows linearly with time.

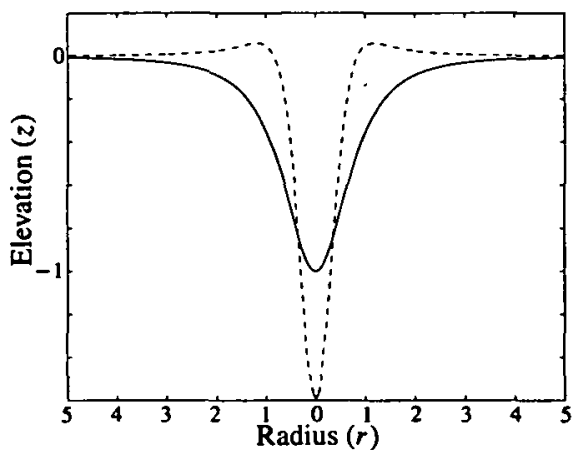

FIGURE 2. Surface deflection coefficients $\eta_{1}(r)$ (solid) and $\eta_{2}(r)$ (dashed) for the single sink case, with initial condition (2.6).

The second-order kinematic condition (3.5) yields

$$
\eta_{2}(r)=\frac{1}{2} \phi_{12}(r, 0)-\frac{1-2 r^{2}}{\left(r^{2}+1\right)^{4}}
$$

and hence we must determine $\phi_{1 z}(r, 0)$.

Using the first-order dynamic condition from (3.3), we obtain

$$
\phi_{1}(r, 0)=-\frac{1}{2}\left(\phi_{0 r}^{2}+\phi_{0 z}^{2}\right)=-\frac{1}{2} \frac{1}{\left(r^{2}+1\right)^{2}} .
$$

This condition and the Laplace equation for $\phi_{1}$ is solved by means of the Hankel transform to give

$$
\phi_{1}(r, z)=\int_{0}^{\infty} e^{x z} J_{0}(x r) x\left[\int_{0}^{\infty} \phi_{1}(s, 0) J_{0}(x s) s d s\right] d x .
$$


However, it is $\phi_{1 z}(r, 0)$ we seek; differentiating under the integral and letting $z=0$ gives

$$
\begin{aligned}
\phi_{1 z}(r, 0) & =-\frac{1}{2} \int_{0}^{\infty} x^{2} J_{0}(x r)\left[\int_{0}^{\infty} \frac{J_{0}(x s) s}{\left(s^{2}+1\right)^{2}} d s\right] d x \\
& =-\frac{1}{8} \int_{0}^{\infty} x^{3} J_{0}(x r) K_{1}(x) d x \\
& =-\frac{1}{8} \frac{k^{3}}{r^{3}}\left(\left(7-8 k^{2}\right) E_{2}(k)-4\left(1-k^{2}\right) E_{1}(k)\right),
\end{aligned}
$$

where $k=r / \sqrt{r^{2}+1}$, and $E_{1}(k), E_{2}(k)$ (sometimes denoted as $F(k)$ and $E(k)$, respectively) are the complete elliptic integrals of the first and second kinds respectively. In the above, the inner integral on the first line was evaluated using Gradshteyn and Ryzhik [8, page 686]. Here, $K_{1}$ is the modified Bessel function of the second kind of first order. The final integral comes from Prudnikov et al. [23, page 366].

We now have a closed form for $\eta_{2}$, shown as a dashed line in Figure 2. Note that this term reinforces the first-order dip. We expect that higher order terms might act to oppose this initial dip.

3.3. Image sink case In this section we assume there is an image sink situated above the free surface, thus satisfying initial condition (2.7), so that

$$
\phi_{0}(r, z)=\frac{1}{\left(r^{2}+(z+1)^{2}\right)^{1 / 2}}+\frac{1}{\left(r^{2}+(z-1)^{2}\right)^{1 / 2}} .
$$

In fact this imposes a condition of no vertical component of velocity at $t=0$ on $z=0$, as if a rigid lid was on the free surface and then instantaneously removed. This turns out to provide a simulation very similar to results of any case in which the initial flow was turned on, allowed to approach a steady state, and then the withdrawal rate increased to the level of interest. The first kinematic condition gives $\eta_{1}(r)=0$, since the initial vertical velocity of the free surface is everywhere zero.

The second kinematic condition gives $\eta_{2}(r)=\phi_{1 z}(r, 0)$, and the first-order dynamic condition gives

$$
\phi_{1}(r, 0)=-\frac{2 r^{2}}{\left(r^{2}+1\right)^{3}}=-2\left(\frac{1}{\left(r^{2}+1\right)^{2}}-\frac{1}{\left(r^{2}+1\right)^{3}}\right) .
$$

Again using the Hankel transform and Gradshteyn and Ryzhik [8], we obtain

$$
\phi_{1 z}(r, 0)=-\int_{0}^{\infty} k^{3} J_{0}(x r) K_{1}(x) d x+\frac{1}{4} \int_{0}^{\infty} k^{4} J_{0}(x r) K_{2}(x) d x
$$


which from Prudnikov et al. [23] is evaluated in terms of complete elliptic integrals as

$$
\begin{aligned}
\phi_{1 z}(r, 0)= & \frac{k^{3}}{4 r^{3}}\left(2\left(19-44 k^{2}+24 k^{4}\right) E_{2}(k)-\left(1-k^{2}\right)\left(23-24 k^{2}\right) E_{1}(k)\right) \\
& -\frac{k^{3}}{r^{3}}\left(\left(7-8 k^{2}\right) E_{2}(k)-4\left(1-k^{2}\right) E_{1}(k)\right),
\end{aligned}
$$

again with $k=r / \sqrt{r^{2}+1}$. This gives rise to the second-order profile $z=\eta_{2}(r)$ as in Figure 3. Note that the initial behaviour is for the central region to rise upward due to the low pressure generated on the surface above the sink.

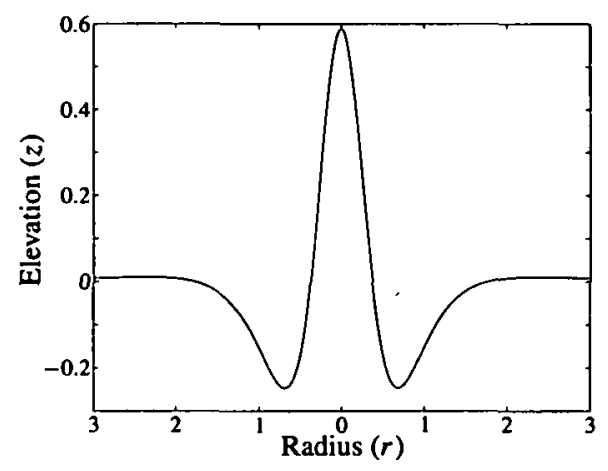

FIGURE 3. Surface deflection coefficient $\eta_{2}(r)$ for the image sink case, with initial condition (2.7).

\section{The numerical method}

We performed a series of simulations using an approach similar to that of Stokes et al. [26] for the two-dimensional line sink case, based on the method described in Scullen and Tuck [25] and Tuck [27]. It is a semi-Lagrangian approach in which we follow the evolution of concentric rings centred above the sink on the free surface subject to an evolving potential. (Radial symmetry guarantees that all points on such a ring have the same elevation.) We use a recursive process to update the global velocity potential and the heights of the $N$ rings on the free surface at each time step. We can specify the position of one such ring by giving its radius and height, thereby reducing the description of the free surface to two parameters.

4.1. Equation formulation Initially, we assume the sample rings are at rest and in some fixed positions $\left(R_{i}^{(0)}, Z_{i}^{(0)}\right)$ on the free surface. We then impose an instantaneous velocity potential function $\phi^{(0)}(r, z)$ that assumes the presence of some sources and/or sinks. 
The recursive step modifies each ring's position and the value of the potential function at it and hence everywhere in the fluid (by solving a Dirichlet problem). Thus if at time step $t$, the $i^{\prime}$ th ring has coordinates $\left(R_{i}^{(t)}, Z_{i}^{(t)}\right)$ and the global velocity potential is $\phi^{(t)}(r, z)$ at all points $(r, z)$ in the fluid, so that $\Phi_{i}^{(t)}=\phi\left(R_{i}^{(t)}, Z_{i}^{(t)}\right)$ is the velocity potential function at the $i$ 'th point on the surface, then the time evolution of all points is given by (dropping the time superscript for simplicity)

$$
\frac{d R_{i}}{d t}=u_{i}, \quad \frac{d Z_{i}}{d t}=v_{i} \quad \text { and } \quad \frac{d \Phi_{i}}{d t}=\frac{1}{2}\left[\left(u_{i}\right)^{2}+\left(v_{i}\right)^{2}\right]-F^{-2}\left(Z_{i}-\beta T_{i}\right) .
$$

Here $u_{i}=\phi_{r}\left(R_{i}, Z_{i}\right), v_{i}=\phi_{2}\left(R_{i}, Z_{i}\right)$ and $T_{i}$ is a surface tension term computed numerically from the current set of free surface points, using the formula

$$
T(r)=\frac{z^{\prime \prime}+z^{\prime} / r}{\left(1+\left(z^{\prime}\right)^{2}\right)^{3 / 2}},
$$

where $(r, z)$ specifies a ring of points on the free surface and the derivatives are with respect to $r$ following the free surface. (We used finite differences to perform these slope and curvature calculations.) Hence the values of $R_{i}, Z_{i}$ and $\Phi_{i}$ can be evolved forward one time step. The task remains to determine $\phi^{(t+1)}$ from the $\Phi_{i}^{(t+1)}$ values just computed. To do this, we must solve a Dirichlet problem.

Assume radii and elevations $P_{i}=\left(R_{i}, Z_{i}\right)$ for the $N$ free surface rings of points at time step $t$, and values $\Phi_{i}$ for $\phi$ given at each. Choose the ring sink with radius and elevation $\left(\rho_{i}, \zeta_{i}\right)$ just "above" the free surface ring given by $\left(R_{i}, Z_{i}\right)$ and approximately normal to the surface. (The exact means of determination in order to optimise the numerical performance is discussed later.)

The potential at a given point $(r, z)$, due to the sinks one unit above and below the origin are $\Phi_{s_{1}}(r, z)=\left(r^{2}+(z+1)^{2}\right)^{-1 / 2}, \Phi_{s_{2}}(r, z)=\left(r^{2}+(z-1)^{2}\right)^{-1 / 2}$, respectively. Additionally, for the $i^{\text {th }}$ ring sink, the contribution to the total potential at the point $(x, y, z)=(r \cos \alpha, r \sin \alpha, z)$ will be

$$
\Phi_{i}(r, z)=\int_{0}^{2 \pi} \frac{\rho_{i}}{\left[r^{2}+\rho_{i}^{2}+\left(z-\zeta_{i}\right)^{2}-2 r \rho_{i} \cos (\theta-\alpha)\right]^{1 / 2}} d \theta,
$$

multiplied by a strength factor to be determined.

Each such integral can be expressed as a linear combination of elliptic integrals of the first and second kinds, and evaluated using standard series expansions.

Thus we assume a series that satisfies Laplace's equation everywhere within the fluid, of the form

$$
\phi(r, z)=\sum_{i=1}^{N} q_{i} \Phi_{i}+\Phi_{s_{1}}+\delta_{1} \Phi_{s_{2}} .
$$

Here, $\delta_{1}$ is 0 or 1 (or to repeat the results of Miloh and Tyvand [21], $\delta_{1}=-1$ ). The source strengths $q_{i}$ are unknown constants that are determined once the values of $\phi$ 
are given at $N$ distinct points, from which we obtain $N$ linear equations in the $q_{i}$ and solve to give $\phi(r, z)$.

Differentiating under the integral signs in the previously obtained expression for $\phi$ allows us to compute $u=\phi_{r}$ and $v=\phi_{2}$, which again can be readily computed in terms of elliptic integrals. This allows us to compute the $u_{i}$ and $v_{i}$ at time $t+1$.

4.2. Computational details We followed Scullen and Tuck [25] and Tuck [27] in distributing the discrete points along the free surface with even spacing near $r=0$ and a geometric increase in spacing beyond a certain point. The sink locations were chosen very much as in [26]. It was found that including a point sink above the surface at $r=0$ caused numerical instabilities, and so the smallest radius ring sink was placed at $r=\delta r / 2$, where $\delta r$ was the local surface point separation distance. It was found that reliable simulations could be obtained if the distance of the ring sinks above the free surface was chosen to be double the local grid spacing, that is, $2 \delta r$. Splines were used to re-position points along the free surface according to the original $R$-spacings, using an arclength-type calculation as described in [26].

The combination of a wide computational domain and small time steps was sufficient to ensure reliable, converged results. Typically, a time step of $\delta t=F / 20$ was adequate for simulations with no surface tension $(\beta=0)$. However, the introduction of even a modest amount of surface tension necessitated a large reduction in step size, for example with $\beta=0.05, \delta t=F / 300$ was required. The difference was due to the appearance of the second derivative in the surface tension term. If the space step $\delta r$ was made too small relative to the time step, the solutions began to exhibit some numerical instability, so the space increment $\delta r=0.025$ was used in most cases. (Recall that the sink depth is one unit.) The values of $N$, the total number of points in the computational domain, and $N_{1}$, the number of evenly spaced points before the geometric expansion outward, never exceeded 200 and 100 respectively.

A Newton iteration scheme was developed based on this distributed sink technique, in order to generate steady solutions. If the number of discrete points used to represent the surface was $n$, the $z$-coordinates and the coefficients $q_{i}$ of the sink terms were used as unknowns at fixed values of $x$. The equations to be satisfied are the steady components of Bernoulli's equation, (2.2), and the kinematic condition, (2.3), both to be satisfied at each of the $n$ points. Laplace's equation is satisfied by the choice of the basis functions. This gave $2 n$ equations in $2 n$ unknowns, and the usual iterative Newton scheme was implemented. On most occasions, the starting values of the sink strengths were set to zero and the surface height was set at $z=0$.

We used Fortran 77 to perform the simulations and then viewed the results using MATLAB. The result was a series of "movies" of the evolution of the free surface in cross-section. 


\section{Results of numerical experiments}

5.1. The single sink case Here we used initial condition (2.6). Regardless of Froude number $F$ and surface tension coefficient $\beta$, the free surface was always initially pulled downwards at $r=0$ by an amount having magnitude of order $O(F)$, in excellent agreement with the small time expansion. If $F$ was large enough the surface would draw down, and at smaller values it would rebound: see Figure 1.

In the zero surface tension case, for $F \geq 0.28$, there was a direct drawdown of the free surface into the sink, with the central point leading the way. For $F \leq 0.27$, the free surface rebounded as a precursor to either a gradual movement to a steady state or perhaps to some unsteady flow, possibly involving breaking waves (see Figure 4). The work of Miloh and Tyvand [21] and Xue and Yue [32] gives qualitatively similar results, but the critical values are lower.

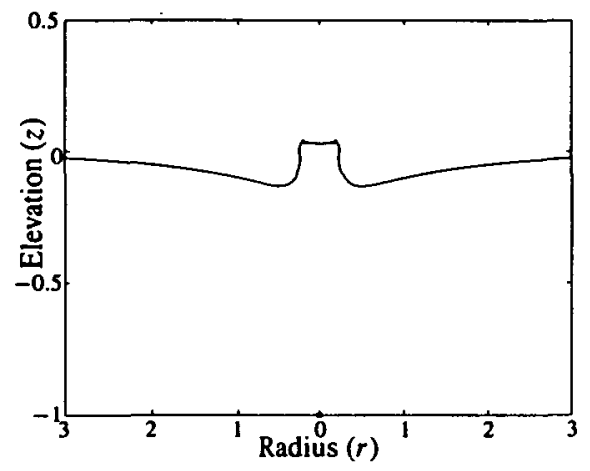

FIGURE 4. Single sink case with $F=0.22, \beta=0, t=0.58$ just before breakdown, showing small breaking waves.

The picture was not changed radically with the introduction of surface tension, although the critical drawdown value increased with $\beta$, and for subcritical $F$ the simulations generally ran longer and moved more clearly towards steady states due to the smoothing effect of the surface tension.

The lowest curve on Figure 5 shows the critical value of $F$ for drawdown as a function of $\beta$ (solid line), indicating a smooth increase with increasing surface tension, similar to what was found in the line sink case [26]. (Figure 5 also shows results for the image sink case discussed below.)

An interesting sidelight to these results is that there appear to be two critical depths. If (and only if) no point on the surface dropped below $z=-0.5$, drawdown was averted regardless of $\beta$. In addition, if the level of the free surface did not drop below $z \approx-0.25$, then the central point was always the global minimum, while if the central point dropped below this point, then the global minimum would occur away from the 


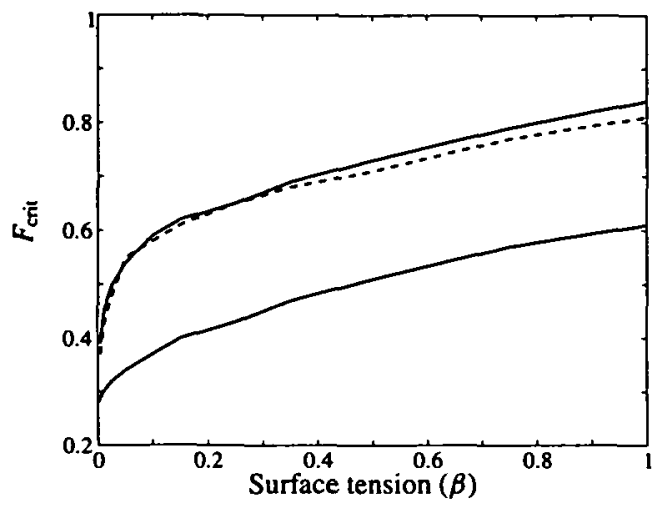

FIGURE 5. Critical Froude number, $F$, against surface tension, $\beta$, for the ramped up image sink case, from simulations (solid top curve-minimal $F$ to guarantee drawdowns), dashed curve maximal $F$ for possible steady states from Newton's method, and the single sink critical Froude number, $F_{1}$ (lowest curve).

centre as other points continued to drop after the centre had rebounded.

5.2. The image sink case Here we used initial condition (2.7). In all cases, there was an initial upward movement of the central point of size $O\left(F^{2}\right)$, and a dip in the surface at around $r=1$. For $\beta=0$, agreement with the small time expansion was good for sufficiently small time.

The picture regarding drawdown was more complex. There appeared to be two kinds of drawdown, direct and indirect. In the indirect drawdown case, a ring of points offset from the origin would draw down first, with a cigar-shaped region directly above the point sink being pinched off: see Figure 6 (a). Such indirect drawdowns occurred for higher values of the Froude number.

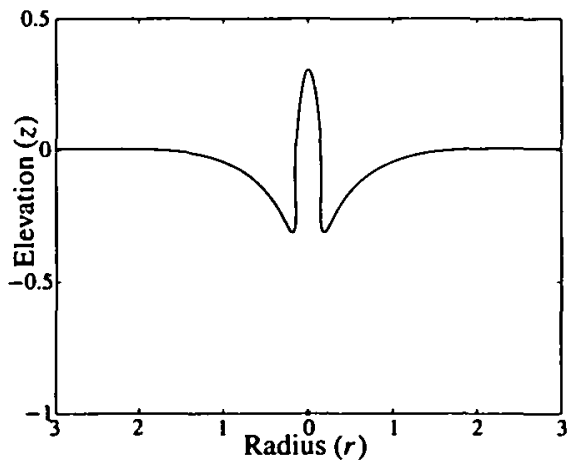

(a) $F=0.78, t=0.88$

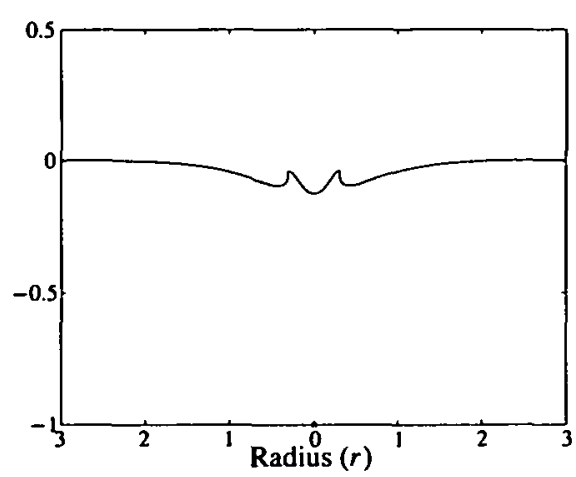

(b) $F=0.38, t=0.85$

FIGURE 6. Image sink case: two profiles with $\beta=0$ and different large $F$. 
For $\beta=0$, the value $F=0.38$ seemed significant. Above this value, the free surface was still descending at the moment the simulations broke down, suggesting a drawdown was likely, and the higher the Froude number, the more likely it appeared that this drawdown would be indirect (Figure 6 (a)). At around $F=0.38$, the central point slowed to a stop before the breakdown, and there was no sign of a continued descent anywhere along the surface, with a breaking wave apparently the cause of the breakdown of the numerical scheme (Figure $6(\mathrm{~b})$ ). Below the value $F \approx 0.38$, the simulations ran for progressively longer periods before breakdown (due to reflections from the boundary of the computational domain), and for sufficiently low values of $F$ would settle toward a steady state. Such steady states were computed using Newton's method up to $F \leq 0.32$. Figure 7 shows a comparison between them and the simulations at (a) $F=0.14, t=1.5$ and (b) $F=0.07, t=1.5$. The simulation in case (b) is very close to the steady solution, and even (a) looks likely to reach the steady solution once the few remaining small waves propagate away. As $F$ increased, it became more difficult to decide what would happen.

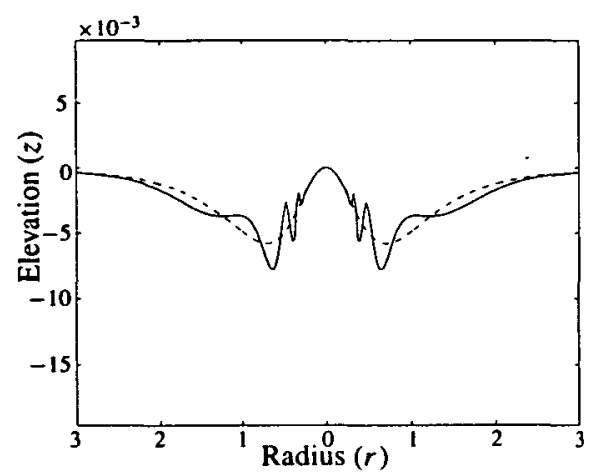

(a) $F=0.14, t=1.5$

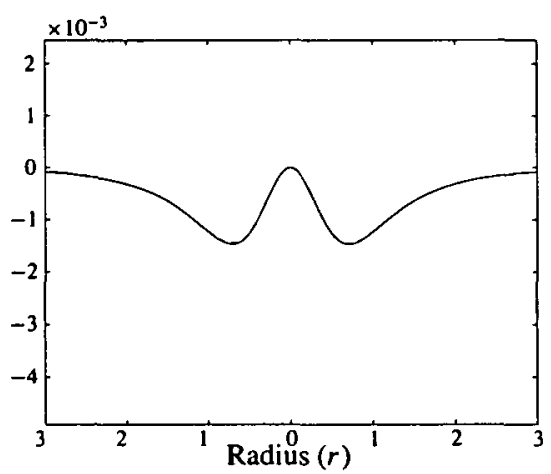

(b) $F=0.07, t=1.5$

FIGURE 7. Image sink case: two profiles with $\beta=0$ and different small $F$. The dashed lines are known steady solutions.

Now it is known that steady states exist up to $F \approx 0.50$ : see [2]. This suggests that at least some of those steady solutions may be unstable flows, or simply cannot be reached as the evolution of a sink flow. Alternatively, it could be that after a transient phase in which there may be breaking waves, the flow will approach a steady flow right up to $F=0.50$.

The introduction of moderate to high amounts of surface tension made things much clearer, with critical values for the two kinds of drawdown becoming clearly defined, and movement to a steady state appearing likely for $F$ below the lower of these critical values. For surface tension $\beta \geq 0.05$, steady solutions (calculated using Newton's method) appeared to exist right up to and slightly beyond the critical drawdown 
values. For example, for $\beta=0.1$, movement to a steady state appeared to occur for $F<0.54$, a direct drawdown appeared to occur for $0.54 \leq F<1.15$, and an indirect drawdown involving the pinching off of a cigar-shaped region occurred for greater Froude numbers. Figure 8 shows the free surface profiles around the time of breakdown for $F=1.25,1.15$ and 1.14 respectively, with $\beta=0.1$.

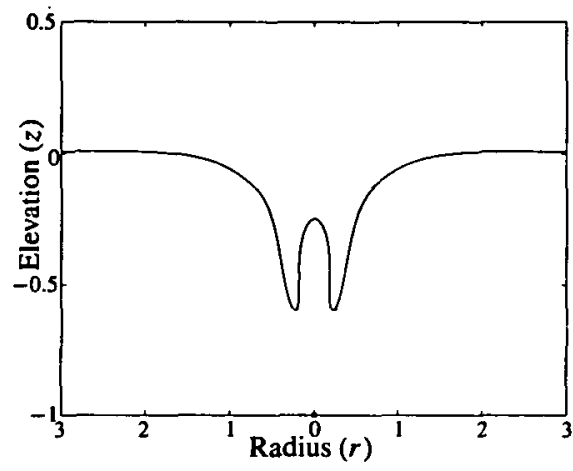

(a) $F=1.25$

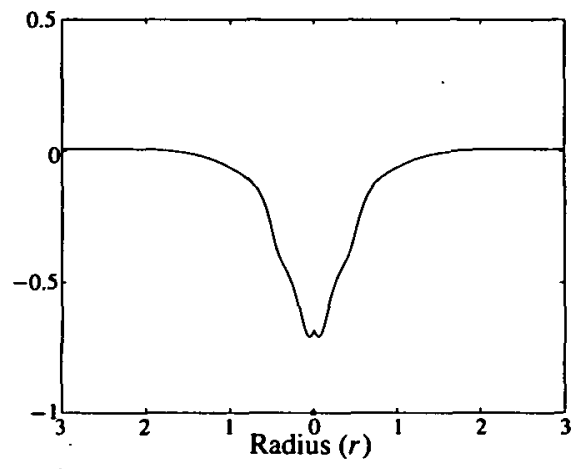

(b) $F=1.15$

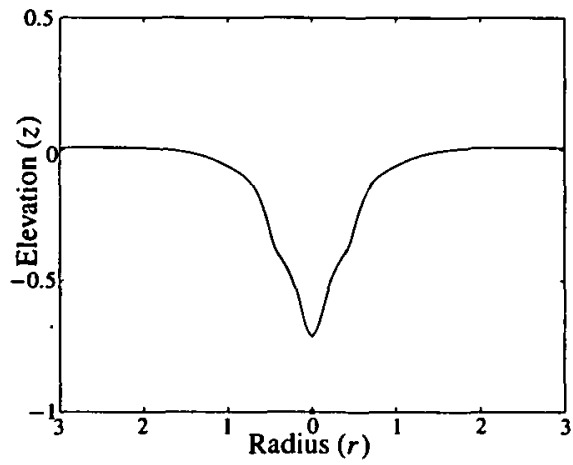

(c) $F=1.14$

FIGURE 8. Image sink case: three profiles near the critical value of $F$ for direct drawdown, $\beta=0.1$.

5.3. Variable flow conditions A series of simulations was carried out in which the flow was initiated at some small value $F_{i}$ (using each of the initial conditions considered above), allowed to reach a quasi-steady state, and then subjected to a large increase in $F$ to $F_{f} \gg F_{i}$. It was clear that such flows behaved almost exactly like the image sink case just discussed with initial condition (2.7), run from $t=0$ and with the final value $F_{f}$ prevailing throughout.

Also considered was an image sink case in which the flow rate was varied, starting at one low Froude number, then slowly increasing up to some final value (corresponding either to a gradual drop in the level of the free surface or a gradual rise in the withdrawal 
rate). The free surface appeared to evolve through the corresponding steady states leading to movement to a steady state over a slightly wider range of (final) Froude numbers. These simulations also produced slightly higher critical drawdown values of $F$, almost completely closing the small gap between critical drawdown value and largest $F$ for which steady states could be found (at least for $\beta \geq 0.05$ ). The resulting critical values of Froude number $F$ are those in Figure 5 (the upper solid curve). The dashed curve indicates the largest value of $F$ for which steady states were found using Newton's method.

\section{Conclusions}

These results are very similar to the line sink situation [26]. In both cases there are at least two effective critical values of Froude number depending on the flow history. Drawdown from an impulsive start depends on the initial dip formed in the surface, but if this is avoided, then the flow will progress for some time (up to a higher Froude number) before eventual drawdown. Coupled with the results of Miloh and Tyvand [21] and Xue and Yue [32], who used different initial conditions, it suggests that flow history is important in understanding the drawdown behaviour, and may be the reason for the many different critical values obtained in experiments. It also suggests that an analysis of the stability of the existing steady-state flows may be very enlightening for understanding the process.

The most intriguing result of this work is that once the solution has approached a steady state, if the flow rate is gradually varied upward, there is an apparent progression through steady-state flows rather than any initiation of significant surface oscillations. This means the existing steady-state solutions [2] provide a very useful representation of the flows.

This has particular relevance to reservoir flows, since withdrawal from a reservoir rarely begins from rest. Thus if the flow ever approaches a steady state, it will henceforth behave like the image sink case, that is, if the flow-rate begins below the drawdown value for a single sink, then the free surface (or interface) will move through the appropriate steady states with disturbances of $O\left(F^{2}\right)$ as the flow rate is adjusted, rather than initiating a new set of $O(F)$ waves. In particular, drawdown will occur at the higher values depicted in Figure 5 rather than the lower values.

\section{References}

[1] A. Craya. "Theoretical research on the flow of nonhomogeneous fluids", La Houille Blanche 4 (1949) 44-55.

[2] L. K. Forbes and G. C. Hocking, "Flow caused by a point sink in a fluid having a free surface", J. Austral. Math. Soc. Ser. B 32 (1990) 233-252. 
[3] L. K. Forbes and G. C. Hocking, "Flow induced by a line sink in a quiescent fluid with surfacetension effects", J. Austral. Math. Soc. Ser. B 34 (1993) 377-391.

[4] L. K. Forbes and G. C. Hocking, "The bath-plug vortex", J. Fluid Mech. 284 (1995) 43-62.

[5] L. K. Forbes and G. C. Hocking, "On the computation of steady axi-symmetric withdrawal from a two-layer fluid", Computers and Fluids 32 (2003) 385-401.

[6] L. K. Forbes, G. C. Hocking and G. A. Chandler, "A note on withdrawal through a point sink in fluid of finite depth", J. Austral. Math. Soc. Ser. B 37 (1996) 406-416.

[7] P. Gariel, "Experimental research on the flow of nonhomogeneous fluids", La Houille Blanche 4 (1949) 56-65.

[8] I. S. Gradshteyn and I. M. Ryzhik, Tables of integrals, series and products (Academic Press, New York, 1965).

[9] D. R. F. Harleman and R. E. Elder, "Withdrawal from a two-layer stratified flow", Proc. ASCE 91 (1965) HY4.

[10] G. C. Hocking, "Cusp-like free-surface flows due to a submerged source or sink in the presence of a flat or sloping bottom", J. Aust. Math. Soc. Ser. B 26 (1985) 470-486.

[11] G. C. Hocking, "Withdrawal from two-layer fluid through line sink", J. Hydr. Engng ASCE 117 (1991) 800-805.

[12] G. C. Hocking, "Supercritical withdrawal from a two-layer fluid through a line sink", J. Fluid Mech. 297 (1995) 37-47.

[13] G. C. Hocking and L. K. Forbes, "A note on the flow induced by a line sink beneath a free surface", J. Aust. Math. Soc. Ser. B 32 (1991) 251-260.

[14] G. C. Hocking and L. K.Forbes, "Supercritical withdrawal from a two-layer fluid through a line sink if the lower layer is of finite depth", J. Fluid Mech. 428 (2001) 333-348.

[15] G. C. Hocking, J.-M. Vanden-Broeck and L. K. Forbes, "Withdrawal from a fluid of finite depth through a point sink", ANZIAM J. 44 (2002) 181-191.

[16] D. G. Huber, "Irrotational motion of two fluid strata towards a line sink", J. Engng. Mech. Div. Proc. ASCE 86 (1960) 71-85.

[17] J. Imberger and P. F. Hamblin, "Dynamics of lakes, reservoirs and cooling ponds", Ann. Rev. Fluid Mech. 14 (1982) 153-187.

[18] G. H. Jirka and D. S. Katavola, "Supercritical withdrawal from two-layered fluid systems. Part 2. Three-dimensional flow into a round intake", J. Hyd. Res. 17 (1979) 53-62.

[19] G. A. Lawrence and J. Imberger, "Selective withdrawal through a point sink in a continuously stratified fluid with a pycnocline", Technical Report No. ED-79-002, Dept. of Civil Eng., University of Western Australia, Australia, 1979.

[20] B. T. Lubin and G. S. Springer, "The formation of a dip on the surface of a liquid draining from a tank", J. Fluid Mech. 29 (1967) 385-390.

[21] T. Miloh and P. A. Tyvand, "Nonlinear transient free-surface flow and dip formation due to a point sink", Phys. Fluids A 5 (1993) 1368-1375.

[22] H. Peregrine, "A line source beneath a free surface", Technical Report No. 1248, Math. Res. Centre, University of Wisconsin, Madison, 1972.

[23] A. P. Prudnikov, Y. A. Brychkov and O. I. Marichev, Integrals and series (Gordon and Breach, New York, 1986).

[24] C. Sautreaux, "Mouvement d'un liquide parfait soumis à lapesanteur. Détermination des lignes de courant". J. Math. Pures Appl. 7 (1901) 125-159.

[25] D. Scullen and E. O. Tuck, "Non-linear free-surface flow computations for submerged cylinders", J. Ship Res. 39 (1995) 185-193.

[26] T. E. Stokes, G. C. Hocking and L. K. Forbes, "Unsteady free surface flow induced by a line sink", J. Eng. Math. 47 (2002) 137-160. 
[27] E. O. Tuck, "Solution of nonlinear free-surface problems by boundary and desingularised integral equation techniques", in Proc. 8th Biennial Computational Techniques and Applications Conference (ed. J. Noye et al.), (World Scientific, Singapore, 1997) 11-26.

[28] E. O. Tuck and J.-M. Vanden-Broeck, "A cusp-like free-surface flow due to a submerged source or sink". J. Aust. Math. Soc. Ser. B 25 (1984) 443-450.

[29] P. A. Tyvand, "Unsteady free-surface flow due to a line source", Phys. Fluids A 4 (1992) 671-676.

[30] J.-M. Vanden-Broeck and J. B. Keller, "Free surface flow due to a sink", J. Fluid Mech. 175 (1987) 109-117.

[31] I. R. Wood and K. K. Lai, "Selective withdrawal from a two-layered fluid". J. Hyd. Res. 10 (1972) 475-496.

[32] X. Xue and D. K. P. Yue, "Nonlinear free-surface flow due to an impulsively started submerged point sink", J. Fluid Mech. 364 (1998) 325-347.

[33] C. S. Yih, Stratified flows (Academic Press, New York, 1980). 